\title{
Frequency-Dependent Attentional Modulation of Local Field Potential Signals in Macaque Area MT
}

\author{
Paul S. Khayat, ${ }^{1,2}$ Robert Niebergall, ${ }^{1,3}$ and Julio C. Martinez-Trujillo ${ }^{1,2}$ \\ ${ }^{1}$ Cognitive Neurophysiology Laboratory, Department of Physiology, McGill University, Montréal, Québec H3G 1Y6, Canada, ${ }^{2}$ Research Center in \\ Neuropsychology and Cognition, University of Montreal, Montréal, Québec H3C 3J7, Canada, and ${ }^{3}$ Cognitive Neuroscience Laboratory, German Primate \\ Center, 37077 Goettingen, Germany
}

Visual attention modulates neuronal responses in primate motion processing area MT. However, whether it modulates the strength local field potentials (LFP-power) within this area remains unexplored, as well as how this modulation relates to the one of the neurons' response. We investigated these issues by simultaneously recording LFPs and neuronal responses evoked by moving random dot patterns of varying direction and contrast in area MT of two male monkeys (Macaca mulatta) during different behavioral conditions. We found that: (1) LFP-power in the $\gamma(30-120 \mathrm{~Hz})$, but not in the $\delta(2-4 \mathrm{~Hz}), \theta(4-8 \mathrm{~Hz}), \alpha(8-12 \mathrm{~Hz}), \beta_{1}(12-20 \mathrm{~Hz})$, and $\beta_{2}(20-30 \mathrm{~Hz})$ frequency bands, was tuned for motion direction and contrast, similarly to the neurons' response, (2) shifting attention into a neuron's receptive field (RF) decreased LFP-power in the bands below $30 \mathrm{~Hz}$ (except the $\theta$ band), whereas shifting attention to a stimulus motion direction outside the RF had no effect in these bands, (3) LFP-power in the $\gamma$ band, however, exhibited both spatial- and motion directiondependent attentional modulation (increase or decrease), which was highly correlated with the modulation of the neurons' response. These results demonstrate that in area MT, shifting attention into the RFs of neurons in the vicinity of the recording electrode, or to the direction of a moving stimulus located far away from these RFs, distinctively modulates LFP-power in the various frequency bands. They further suggest differences in the neural mechanisms underlying these types of attentional modulation of visual processing.

\section{Introduction}

The frequency of action potentials fired by neurons in area middle temporal (MT) of monkeys encodes the contrast, direction, and speed of moving stimuli (Zeki, 1980; Felleman and Kaas, 1984; Sclar et al., 1990). The firing rate of these neurons is also modulated when attention is directed into their receptive fields (RFs), or to the motion direction of a stimulus outside the RF (Treue and Maunsell, 1996; Seidemann and Newsome, 1999; Treue and Martínez Trujillo, 1999). A previous study has demonstrated that the amplitude of local field potentials oscillations (LFP-power) in this area encodes, within certain frequencies, motion direction and speed (Liu and Newsome, 2006). However, it remains uninvestigated whether directing attention to an object's spatial position, or feature encoded by MT neurons, modulates LFP-power in this area.

LFPs contain oscillations with frequencies below $200 \mathrm{~Hz}$ that can be recorded from the same electrode as higher frequency spikes fired by single units. LFPs are thought to represent synaptic activity within a local network (in the recorded cell's vicinity), as well as voltage-dependent membrane oscillations, spike components and afterpotentials, and inputs from other brain regions

Received Jan. 24, 2010; revised March 30, 2010; accepted April 9, 2010.

This study was supported by a Natural Sciences and Engineering Research Council of Canada Fellowship awarded to P.S.K., and Canada Foundation for Innovation and Canadian Institutes of Health Research grants awarded to J.C.M.-T. We thank W. Kucharski and S. Nuara for technical assistance.

Correspondence should be addressed to Paul S. Khayat, Department of Physiology, McGill University, Room 1220, 3655 Prom. Sir W. Osler, Montréal, QC H3G 1Y6, Canada. E-mail: paul.khayat@mcgill.ca.

DOI:10.1523/JNEUROSCI.0404-10.2010

Copyright $\odot 2010$ the authors $\quad 0270-6474 / 10 / 307037-12 \$ 15.00 / 0$
(Mitzdorf, 1985, 1987; Kruse and Eckhorn, 1996; Buzsáki, 2002; Logothetis, 2003; Logothetis and Wandell, 2004; Katzner et al., 2009; Khawaja et al., 2009). Understanding how LFP-power in area MT relates to changes in stimulus attributes such as contrast and direction, and how it is influenced by visual attention may reveal important aspects of the computations underlying sensory and cognitive processing in the primate brain.

In other visual areas than MT, previous studies have reported that LFP-power in the $\gamma$ frequencies $(>25 \mathrm{~Hz})$ is selective to stimulus attributes (Frien et al., 2000; Kayser and König, 2004; Henrie and Shapley, 2005; Berens et al., 2008; Katzner et al., 2009). In area V4, spatial attention enhances $\gamma$-band power, while it generally decreases power in lower frequencies (Fries et al., 2001, 2008; Taylor et al., 2005). In area V1, however, Lakatos et al. (2008) reported that during certain tasks attention could increase LFP-power in the $\delta$ band $(<4 \mathrm{~Hz}$ ) (see also Schroeder and Lakatos, 2009). These attentional effects in different bands may be a generalized finding across visual cortical areas that reflects the local and/or global neural computations underlying attentional filtering of behaviorally relevant signals (Fries, 2009). If this is the case, we should find frequency-dependent modulations of LFPs recorded from area MT during attentional tasks.

Here, we explored these issues by measuring LFP-power and spiking activity evoked by moving random dot patterns with various directions and contrasts in area MT of macaques during different experimental conditions. First, we determined the direction and contrast selectivity of the LFPs in different frequency bands, as well as the correlation of the LFP-power in each band with the neurons spiking activity. Second, we isolated in each 
band the effects of attending to an object's spatial position (inside vs outside a neuron's RF), or to a feature encoded by MT neurons (motion direction), by contrasting measurements corresponding to the different conditions.

\section{Materials and Methods}

Two male macaque monkeys (Macaca mulatta) participated in the experiments. All procedures complied with the Canadian Council of Animal Care guidelines and were approved by the McGill University animal care committee. Standard surgical and electrophysiological techniques were used to record neuronal activity in area MT (Khayat et al., 2010).

Behavioral task. On each trial, the animal had to press a button and fixate within a circular window of $1.5^{\circ}$ diameter centered on a small fixation spot ( $0.06^{\circ}$ square). After $470 \mathrm{~ms}$, two pairs of moving RDPs appeared, one located inside the RF of the recorded MT neuron, and the other located outside, in the opposite hemifield (Fig. 1). Each pair consisted of a high contrast RDP moving in the neuron's antipreferred direction (AP-pattern) and a test RDP (testpattern) that: (1) had the same contrast as the AP-pattern but moved, from trial to trial, in different directions (direction configuration), or (2) moved in the neuron's preferred direction but had, from trial to trial, different contrasts (contrast configuration).

Three different task conditions were used. In the fixation condition (Fig. $1 A$ ), the animal detected a subtle luminance change in the fixation spot, which occurred at a random interval between 1010 and 3250 $\mathrm{ms}$ after stimulus onset. Here, the animal was required to ignore changes in the direction of any of the RDPs. In the other conditions (Fig. $1 B$ ), a small line $\left(1^{\circ}\right.$ length) appeared next to the fixation spot $350 \mathrm{~ms}$ after stimulus onset. This cue-line pointed toward one of the AP-patterns, thereby instructing the monkey to direct attention to this pattern located either inside (attend-AP in), or outside (attend-AP out) the RF. After a variable delay of 660-2900 $\mathrm{ms}$ from cue onset, the target (i.e., cued AP-pattern) underwent a brief direction change (23 during $100 \mathrm{~ms}$ ). The animal had to release the button within a response time interval of $150-500 \mathrm{~ms}$ after the change to receive a juice reward.

In the two attentional conditions of this task (attend-AP in/out), attention is always on a high contrast pattern moving in the antipreferred direction (AP-pattern). This avoided the possibility that the animal's performance would substantially change while manipulating the direction or contrast of the test-pattern across trials. Also, by using a combination of antipreferred + test-pattern, we could obtain a modulation of the firing rate that ranges from very low (i.e., close to spontaneous response evoked by the combination of the AP-pattern with the $90^{\circ}$ away direction, or lowest contrast test-pattern) to $\sim 70-80 \%$ of the response to the preferred direction (elicited by the combination of AP-pattern and preferred test-pattern direction) (Martínez-Trujillo and Treue, 2002; Khayat et al., 2010). This allows us to explore direction and contrast tuning as well as the effect of attention within this $70-80 \%$ response range.

To ensure that during the attend-AP in and out conditions the monkey was focusing attention on the target, on half of the corresponding trials the uncued AP-pattern (the distracter), located in the opposite visual hemifield, briefly changed direction. The monkey had to ignore this distracter change and wait until the target changed. Trials in which the monkey responded to the distracter change, or broke fixation before the target's change occurred were terminated without reward and considered errors. The different trial types were presented in random sequence, and both animals performed between 6 and 15 trials (median $=12$ trials) per stimulus type in each behavioral condition. Only correctly performed trials, with no change events within the analyzed period, were included in the analysis.

By comparing responses between the different conditions, we could isolate the effects of: (1) spatial attention (attend-AP out vs attend-AP in: directing attention from the AP-pattern outside the RF to the AP-pattern inside). Here, the potential target stimuli (AP-patterns inside and outside the RF) differ in their spatial positions, but they share the same feature (motion direction), which equates the effect of directing attention to this stimulus feature; (2) directing attention to a motion direction (fixation vs attend-AP out: directing attention from the fixation condition, where no motion direction is attended, to the pattern moving in the cell's antipreferred direction outside the RF). Here, the RF stimuli are unattended, as the focus of attention remains outside the RF. However, attention is directed from a neutral condition to the antipreferred direction; (3) spatial attention and attending to a motion direction (fixation vs attend-AP in: directing attention from the fixation condition, where no motion direction is attended, to the pattern moving in the cells antipreferred direction inside the RF). Here, attention is directed into the RF and to the neuron's antipreferred direction. These experimental manipulations have proven to be useful at isolating the effects of attending into the RF, or to a motion direction, on neuronal activity in area MT (Treue and Martínez Trujillo, 1999; Martinez-Trujillo and Treue, 2004; Khayat et al., 2010).

Stimuli. The stimuli were back-projected on a screen by a video projector (NEC WT610, $1024 \times 768$ pixels resolution, $85 \mathrm{~Hz}$ ). The animals viewed the screen at a distance of $57 \mathrm{~cm}$. The RDPs were generated by plotting bright dots on a dark background with a density of 4 dots per degree $^{2}$ within a circular stationary virtual aperture. All dots within one RDP moved coherently at the preferred speed of the recorded neuron $($ median $=11 \%$ s; range $=4-32 \%$ ), and were replotted at the opposite side when they crossed the border of the aperture. The size of the RDP (1.3- $3^{\circ}$ diameter) was chosen so that the two patterns fit inside the boundaries of the classical RF excitatory region. Stimulus contrast was measured as the SD of luminance values (Martínez-Trujillo and Treue, 
2002; Khayat et al., 2010) and expressed in percentage of the highest value. In the contrast configuration, we used different contrast levels of the test-pattern $(0.02,0.1,0.3,0.7,1.5,14$ and $100 \%$ contrast $)$ relative to the contrast level of the AP-pattern. In the direction configuration, we used 7 different motion directions of the test-pattern, from the recorded neuron's preferred direction in steps of $15^{\circ}$ until $90^{\circ}$ away (Fig. $1 \mathrm{~A}$ ) (Khayat et al., 2010).

Recordings and data analysis. Transdural penetrations were made with stainless steel guide tubes $(0.3-0.5 \mathrm{~mm}$ diameter) through a chamber implanted on top of a craniotomy of the parietal bone that provided access to area MT (Khayat et al., 2010). Spikes and LFPs were recorded simultaneously using standard epoxy-insulated extracellular tungsten electrodes (FHC Inc.; impedance $=1-2 \mathrm{M} \Omega$ at $1 \mathrm{kHz}$ ), with the guide tube (impedance $<0.1 \Omega$ ) serving as the reference. We used a Plexon data acquisition system to record and store the neural data (Plexon Instruments). The electrode signal was passed through a headstage with unit gain and then split to separately extract the spike and the LFP components. For the LFPs, the signal was filtered (through hardware filters) between 0.7 and $170 \mathrm{~Hz}$, before being amplified and digitized at $1 \mathrm{kHz}$. For spike recordings, the signal was filtered between 250 and $8000 \mathrm{~Hz}$, amplified and digitized at $40 \mathrm{kHz}$. Single-unit spiking activity was then isolated using a window discriminator.

In each recording session, we used an interactive stimulus presentation program and online display of spiking activity to qualitatively assess the recorded neuron's RF location, size, and determine its preferred motion direction and speed. Cells were determined to be from MT according to their response properties (directionality and RF position and size), and to the position of the electrode relative to the superior temporal sulcus assessed through MRI images (Khayat et al., 2010). All recorded neurons had RFs located contralateral to the recording hemisphere/sites and we observed a preponderance of RFs centered between $7^{\circ}$ and $12^{\circ}$ from the fixation spot in the lower quadrant and horizontal meridian. During the recordings, an infrared eye-tracking device (EyeLink) was used to monitor eye position at a sampling frequency of $200 \mathrm{~Hz}$.

The off-line analysis of the LFP signals was conducted using Matlab software (MathWorks). In each trial, the raw signal was subdivided into several frequency bands (see below) using a second-order, bidirectional, zero phase Butterworth filter, and full-wave rectified. Each rectified bandpass filtered signal was then averaged within two different epochs of $500 \mathrm{~ms}$ allowing a spectral resolution of $2 \mathrm{~Hz}$ : a baseline period (from -470 to $30 \mathrm{~ms}$ relative to stimulus onset), and a response or stimulus presentation time period that started $510 \mathrm{~ms}$ after stimulus onset and ended before the occurrence of a stimulus change. The response analysis period therefore started $160 \mathrm{~ms}$ after cue-onset, to make sure that the animal had time to direct attention to the target (Khayat et al., 2006; Busse et al., 2008). The rectified bandpass filtered LFP-activity reflects the signal amplitude or strength, and corresponds to the square root of the power on each trial, within a given time period and a given frequency band (Leopold et al., 2003). We determined stimulus-related changes in LFP-power for each frequency band and each trial by computing the base-10 logarithm of the ratio between the stimulus period activity and the baseline period activity $\left[\log\right.$-ratio $=\log \left(\right.$ Power $_{\text {stimulus }} /$ Power $\left.\left._{\text {baseline }}\right)\right]$, and then averaging these values across trials and recording sites.

For the stimulus tuning analyses, the signal spectrum between 2 and $170 \mathrm{~Hz}$ was first analyzed with a bandwidth of $2 \mathrm{~Hz}$. Then, we recomputed the power within predetermined frequency bands, using different bandwidth settings. For frequencies lower than $30 \mathrm{~Hz}$, we used the conventions of human electroencephalography (EEG bands): $\delta 2-4 \mathrm{~Hz}, \theta$ $4-8 \mathrm{~Hz}, \alpha 8-12 \mathrm{~Hz}, \beta 12-30 \mathrm{~Hz}$ (Buzsáki, 2006), with the exception that the $\beta$ band was divided into two sub-bands $\left(\beta_{1} 12-20 \mathrm{~Hz}\right.$ and $\beta_{2} 20-30$ $\mathrm{Hz}$ ). For frequencies higher than $30 \mathrm{~Hz}$ ( $\gamma$ and above band), we used successive, nonoverlapping $20-\mathrm{Hz}$-wide bands covering the entire spectrum between 30 and $170 \mathrm{~Hz}$ (Liu and Newsome, 2006). For the attentional modulation analyses, in addition to the different EEG bands below $30 \mathrm{~Hz}$, we pooled the higher frequencies (i.e., successive 20-Hz-wide bands) into one broader band signal by recomputing the power between 30 and $120 \mathrm{~Hz}$ ( $\gamma$ range or band).

To compare the LFP data with the spiking activity, we also determined in each trial the neuron's averaged firing rate during the baseline and
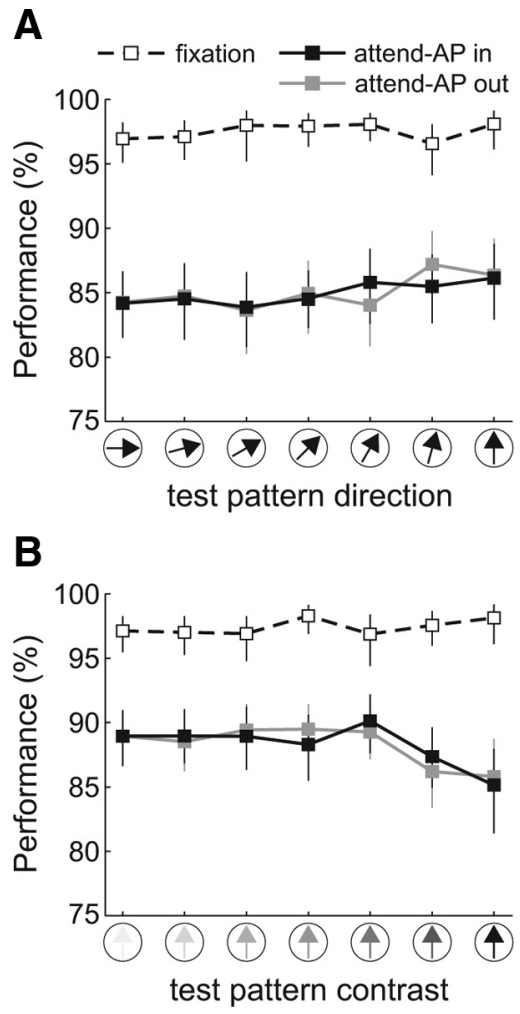

Figure 2. Task performance. $\boldsymbol{A}, \boldsymbol{B}$, Average performance (hit rate) during the fixation (dashed line), attend-AP in (black), and attend-AP out (gray) conditions as a function of the test-pattern direction $(\boldsymbol{A})$ and contrast $(\boldsymbol{B})$. Error bars denote $95 \%$ confidence intervals computed through a bootstrap procedure.

stimulus presentation time periods, and computed the log-ratio. We determined the significance in stimulus-related effects and attentional modulation of LFPs and spiking activity using parametric statistical tests (one-way ANOVA and $t$ test). The effects of stimulus configuration and condition on performance were determined using nonparametric statistics (Kruskal-Wallis ANOVA and sign test).

\section{Results}

\section{Task performance}

We analyzed single-unit firing rate (spiking activity) and LFPs from 81 sites in area MT of two macaques (monkey Se, $n=48$; monkey Lu, $n=33$ ) performing an attention-demanding task. During the task, two pairs of RDPs moving at the neuron's preferred speed were presented, one inside and the other outside the cell's RF. Each pair consisted of a high contrast RDP moving in the neuron's antipreferred direction (AP-pattern) and a test RDP. The latter could have the same contrast as the AP-pattern but moved from trial to trial in different directions (direction configuration), or could move in the neuron's preferred direction but have from trial to trial different contrasts (contrast configuration) (see sketches in Fig. $1 \mathrm{~A}$ ). On each trial, the animals had to either attend to the fixation spot and detect a brief change in its luminance (fixation condition, Fig. $1 A$ ), or to one of the AP-patterns (attend-AP in and attend-AP out condition, Fig. $1 B$ ) and detect a brief change in its motion direction.

Figure 2 shows the performance (hit rate) in the three behavioral conditions, averaged across sessions $(n=81)$ and monkeys, for the different trial types of the direction (Fig. 2A), and contrast (Fig. $2 B$ ) configurations. The averaged performance for each stimulus pair was similar during both attend-AP conditions ( $p>0.2$, sign test), and lower than during the fixation condition $\left(p<10^{-6}\right.$, sign test $)$. 
However, in each condition, performance remained relatively constant across levels of each of the manipulated test parameters (direction and contrast) $(p>0.3$, Kruskal-Wallis nonparametric ANOVA for each condition, with stimulus as a factor), indicating that changes in the direction or contrast of the test-pattern did not significantly influence performance. This allowed us to investigate sensory tuning and attentional modulation of LFPs and spiking activity across different stimulus conditions that yielded similar performance levels (Khayat et al., 2010).

\section{Stimulus tuning of LFP signals}

We investigated whether the LFP signal in different frequency bands was tuned for motion direction and contrast. Figure $3 \mathrm{~A}$ shows examples of raw LFP traces and spike trains simultaneously recorded from the same electrode in area MT during trials with two high-contrast RDPs moving in opposite directions (preferred and antipreferred) presented inside the unit's RF (see top panel).

We first examined the shape of the LFP power spectrum from 2 to $170 \mathrm{~Hz}$ by bandpass filtering the signal with a $2 \mathrm{~Hz}$ frequency resolution (see Materials and Methods). On each trial, the power was determined separately for each $2 \mathrm{~Hz}$ band during the $500 \mathrm{~ms}$ baseline period before stimulus onset, and during the period from 510 to $1010 \mathrm{~ms}$ after stimulus onset (Fig. $3 A$, shaded areas), and then averaged across trials. Figure $3, B$ and $C$, shows the LFP signal power (mean \pm SEM) during fixation trials of the same example site depicted in Figure 3A, and averaged across sites corresponding to each monkey. In all cases, the power was dominated by lowfrequency components, decreasing at higher frequencies during both the baseline (gray), and the stimulus period (black).

To determine stimulus-related changes in LFP-power, we computed in each trial the base- 10 logarithm of the ratio between the power during both time periods $\left[\log _{10}\left(\right.\right.$ Power $_{\text {stimulus }} /$ Power $\left._{\text {baseline }}\right)$ ], and then averaged these values across trials and recording sites (Fig. $3 B, C$, bottom). Observe that this analysis shows the average log-ratio across trials and not the log-ratio of the mean values plotted in the top. The results of the single site example, as well as those averaged across the population of sites of each individual monkey (monkey Se, $n=48$; monkey Lu, $n=33$ ) followed the same profile: stimulus presentation reduced the power at frequencies below $\sim 30 \mathrm{~Hz}$, and increased it at higher frequencies.

We conducted the same analysis for the different stimulus pairs of the direction and contrast configuration (Fig. $4 A, D$ ) by pooling the data from both animals $(n=81)$. Figure 4 illustrates the mean relative power (i.e., log-ratio) across frequencies during fixation trials with different directions (Fig. 4A) and contrasts (Fig. 4D) of the test-pattern. In general, stimulus presentation reduced the power at frequencies below $\sim 30 \mathrm{~Hz}$ (blue colors), and increased it at higher frequencies (red and yellow). Moreover, changing the test-
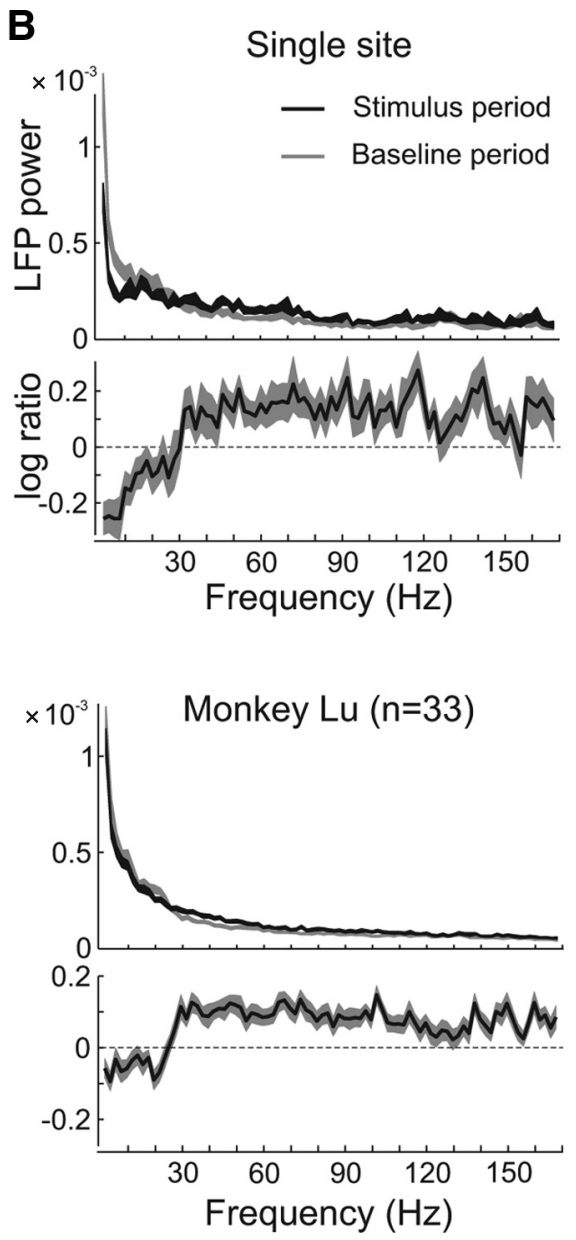

Figure 3. LFP analysis. $\boldsymbol{A}$, LFP activity traces of single fixation trials at an example-recording site. The spike trains of the simultaneously recorded single unit are also shown. The recorded activity is aligned to the onset of the stimulus depicted on top.

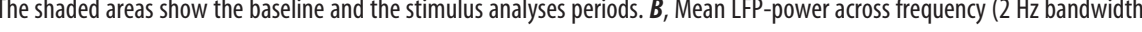
computed as the base-10 logarithm of the ratio between the power in the two analysis periods. C, LFP-power averaged across the recording sites of monkey Se $(n=48)$ and $\mathrm{Lu}(n=33)$ in the two time periods during fixation trials. The relative power (log-ratio, bottom) was computed for each recording site (as in $\boldsymbol{B}$ ) and then averaged across sites.

pattern direction away from the preferred direction or lowering its contrast progressively decreased the magnitude of the relative LFPpower at frequencies above $\sim 30 \mathrm{~Hz}$ ( $\gamma$ range) (Fig. $4 A, D$, red-yellow gradient from right to left), but had not effect at frequencies below $\sim 30 \mathrm{~Hz}$. The results were similar in both animals.

To quantify these observations, we computed for each site the relative LFP-power in predetermined frequency bands. For frequencies above $30 \mathrm{~Hz}$, we used successive 20-Hz-wide frequency bands $(30-50,50-70,70-90,90-110,110-130,130-150,150-$ $170 \mathrm{~Hz}$ ) (Liu and Newsome, 2006). For frequencies below $30 \mathrm{~Hz}$, we used the classical EEG bands: $2-4 \mathrm{~Hz}(\delta), 4-8 \mathrm{~Hz}(\theta), 8-12$ $\mathrm{Hz}(\alpha), 12-20 \mathrm{~Hz}\left(\beta_{1}\right)$, and $20-30 \mathrm{~Hz}\left(\beta_{2}\right)$ (Buzsáki, 2006). Across the population of sites the relative LFP-power in the classical EEG bands did not significantly change with changes in the test-pattern direction (Fig. $4 B$ ) or contrast (Fig. $4 E)(p>0.5$, one-way ANOVA). On the other hand, in the above- $30 \mathrm{~Hz}$ bands, the relative power across sites decreased as the test-pattern's motion direction deviated from preferred (Fig. $4 C$ ), or decreased contrast (Fig. $4 F)(p<0.0005$, one-way ANOVA).

We further measured stimulus selectivity of the LFP signal by computing for each recording site and frequency band the slope 
A Rel. power $-0.2 \quad-0.1 \quad 0 \quad 0.1$

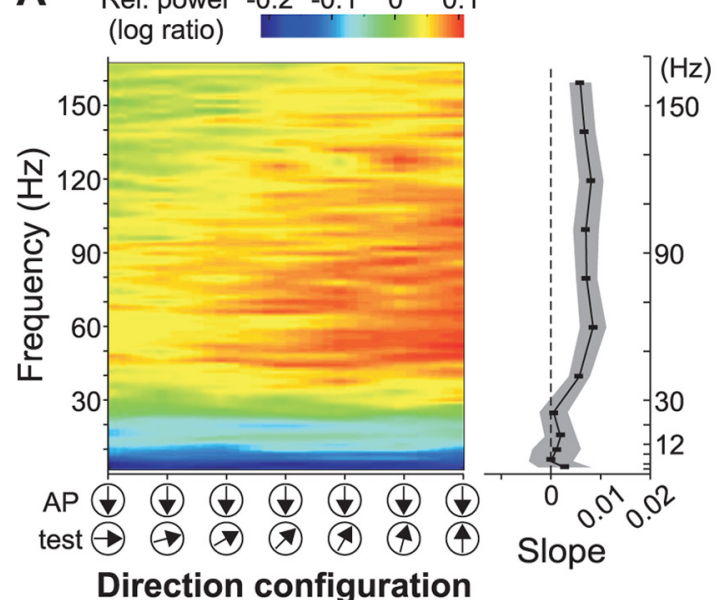

D

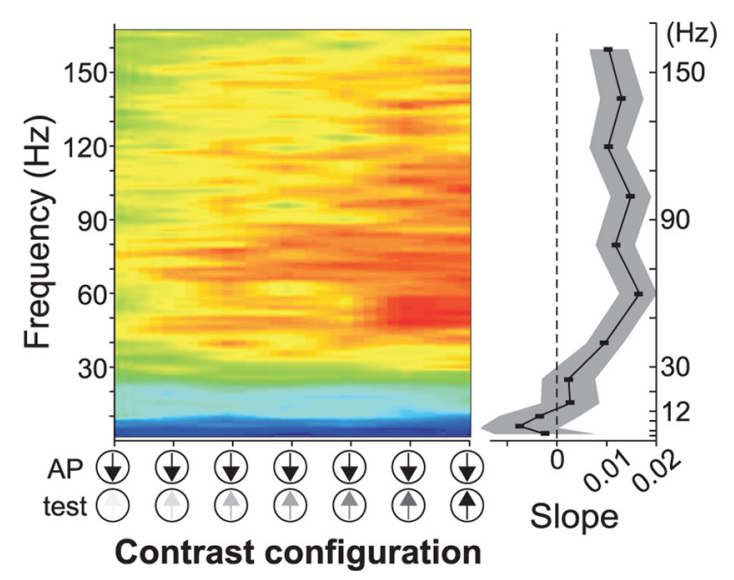

B Frequency bands $<30 \mathrm{~Hz}$

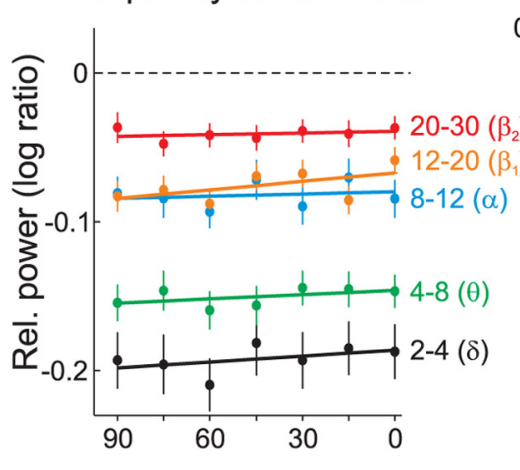

test pattern direction (degrees from preferred)
C

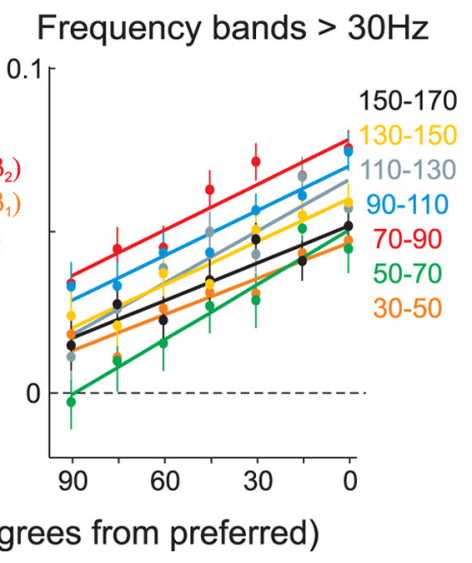

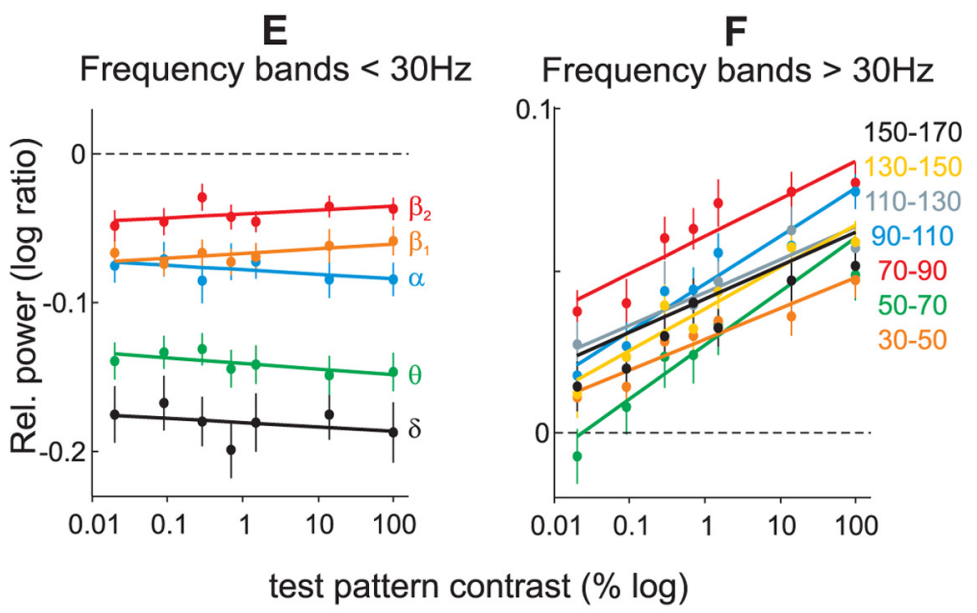

Figure 4. LFP tuning in the direction and contrast configuration. $\boldsymbol{A}$, Color plot showing the relative LFP-power across frequency (2 Hz bandwidth) and test-pattern motion directions during fixation trials averaged across recording sites $(n=81)$. The color represents the change in power during stimulus presentation relative to the baseline activity (log-ratio). For visual display purposes, the data points across frequency and stimulus pairs were interpolated. The graph on the right shows the mean slope ( $95 \%$ confidence interval shown in gray) of a regression line fitted to the data in predetermined frequency bands (see $\boldsymbol{B}$ and $\boldsymbol{C}$, and Results). The slope was computed for each recording site and then averaged across sites. $\boldsymbol{B}, \boldsymbol{C}$, Mean relative LFP-power in different frequency bands below $(\boldsymbol{B})$ and above $(\boldsymbol{C}) 30 \mathrm{~Hz}$, for the population of recording sites $(n=81)$ as a function of the test-pattern motion direction. Error bars, SEM. Note the different scale between the panels. $\boldsymbol{D}-\boldsymbol{F}$, Data in the contrast configuration. Same conventions as above.

of a regression line fitted to the data corresponding to the direction and contrast configurations (see lines in Fig. $4 B, C, E, F$ ). In both configurations, the average slope across recording sites in the below-30 Hz bands was not different from zero $(p>0.05, t$ test; $r^{2}$ range: $\left.0.04-0.5\right)$, indicating that the signal power was not tuned for direction and contrast. On the other hand, for the above-30 Hz bands the average slopes were significantly larger than zero ( $p<10^{-6}, t$ test; $r^{2}$ range: $\left.0.8-0.94\right)$, indicating that the relative power was tuned for both direction and contrast (Fig. $4 A, D$, see right panels).

This LFP-signal tuning in the above- $30 \mathrm{~Hz}$ frequencies parallels the tuning found in the neuronal spiking activity recorded from the same electrode (Fig. 5A). To facilitate the comparison with the LFP data, we determined the neurons relative firing rate by computing the base-10 logarithm of the ratio between the spiking activity during the stimulus and baseline periods in each trial, and then pooling across trials and cells. We then computed the correlation between spikes and LFPs by plotting the relative LFP-power in each frequency band as a function of the relative firing rate, and fitting a regression line to the data. In the direction (Fig. $5 B$, left) and contrast (Fig. $5 B$, right) configurations, the firing rate was significantly and positively correlated with the
LFP-power in the above- $30 \mathrm{~Hz}$ frequency bands $\left(r^{2}\right.$ range: $0.78-$ 0.94; $p<0.005, t$ test $)$, but not in the below-30 Hz bands $\left(r^{2}\right.$, range $0.04-0.3 ; p>0.05$, $t$ test) (Fig. $5 C$, see mean linear regression slopes $\pm 95 \%$ confidence interval, for each band).

\section{Attentional modulation of low-frequency $(<30 \mathrm{~Hz})$ LFP signals}

To examine whether visual attention modulated the LFP signal in our sample of recording sites, we compared the relative LFPpower in the different bands between the three behavioral conditions (fixation, attend-AP in and attend-AP out). Figure 6 shows the relative LFP-power in the $\delta(2-4 \mathrm{~Hz}), \theta(4-8 \mathrm{~Hz}), \alpha(8-12$ $\mathrm{Hz}), \beta_{1}(12-20 \mathrm{~Hz})$, and $\beta_{2}(20-30 \mathrm{~Hz})$ frequency bands averaged across recording sites $(n=81)$ in the three behavioral conditions of the direction and contrast configuration, as well as attentional effects. In none of these lower frequency bands did the magnitude of the LFP-power vary significantly with changes in the test-pattern direction (Fig. 6A, left), or contrast (Fig. 6B, left) $(p>0.5$, one-way ANOVA) when the animals had to attend to the fixation spot (black dashed line, fixation), or directed attention to the AP-pattern inside (black solid line, attend-AP in) or outside (gray solid line, attend-AP out) the RF. However, except 
A
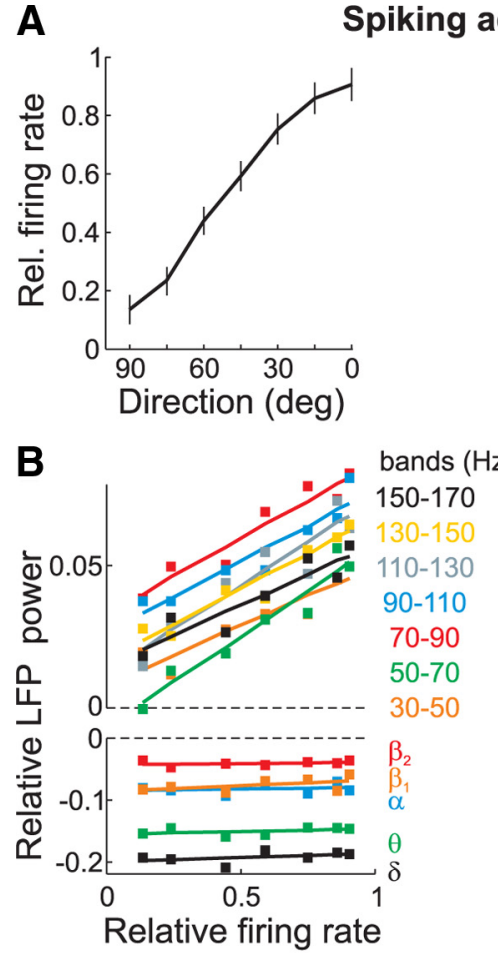

C

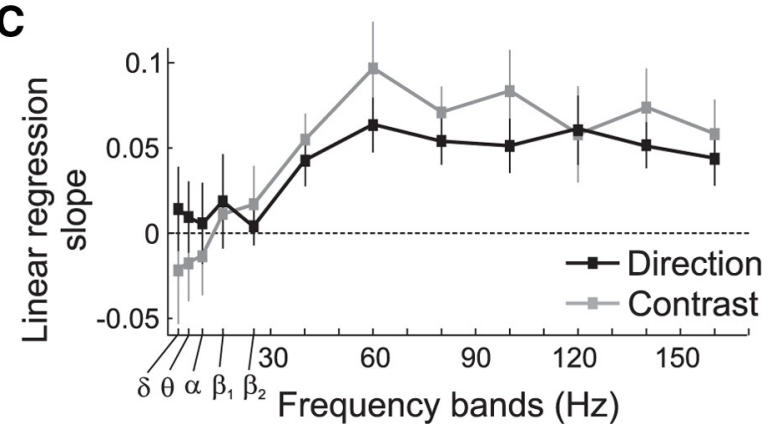

Figure 5. Relationship between LFPs and spiking activity. $\boldsymbol{A}$, Relative firing rate during fixation trials averaged across recording sites $(n=81)$ in the direction (left) and contrast (right) configuration. Error bars, SEM. $\boldsymbol{B}$, Relative LFP-power in the different bands versus the relative firing rate, in the direction (left) and contrast (right) configuration. A regression line was fitted to the data points. $\boldsymbol{C}$, Slope of the linear regression fits shown in $\boldsymbol{B}$ for the different bands, in the direction (black) and contrast (gray) configuration. Error bars, $95 \%$ confidence interval.

for the $\theta$ band, the strength of the LFP-power across all testpattern directions and contrasts appears to be reduced when the animals directed attention inside the $\mathrm{RF}$ (attend-AP in) relative to the other conditions.

We quantified these observations by computing, for each recording site and stimulus combination, the difference in LFPpower between the attend-AP in and attend-AP out or fixation condition, and converting these values to percentages of the LFPpower in the attend-AP out or fixation condition, depending on the comparison to be made $(100 \times$ [attend-AP in - attend-AP out]/attend-AP out $)$, or $(100 \times$ [attend-AP in - fixation]/fixation). In the $\delta, \alpha, \beta_{1}$, and $\beta_{2}$ bands, but not in the $\theta$ band, the difference in the LFP-power pooled across sites (Fig. 6, colored panels) was significantly lower than zero when comparing the attend-AP in and attend-AP out condition (blue), and the attend-AP in and fixation condition (red), for the majority of stimulus pairs (open symbols, $p<0.05, t$ test). The magnitude of this effect extended from $\sim 20 \%$ (in the $\delta$ band) to $\sim 75 \%$ (in the $\beta_{2}$ band) suppression in attend-AP in trials compared with
attend-AP out or fixation trials. This cannot be explained by variations in the animals' behavior, since the task in both attend-AP in and out trials, as well as the animal performance were similar (Fig. 2). Rather, it appears to be caused by directing attention into the RF, because the suppression was equally strong when comparing attend-AP in to fixation, and attend-AP in to attend-AP out, while there was not effect when comparing attend-AP out to fixation. We will further investigate this issue in a separate section (see end of the Results).

\section{Attentional modulation of LFP signals in the $\gamma$ band: direction configuration}

To examine attentional effects in frequency bands above $30 \mathrm{~Hz}$, we determined the relative power in the $\gamma$ band $(30-120 \mathrm{~Hz})$ in the three behavioral conditions. We pooled the data across these frequencies since in our previous analysis the LFP-power was similarly tuned for motion direction and contrast in all bands from 30 to $120 \mathrm{~Hz}$. Additionally, previous studies of attention have described a signal modulation in this frequency range (Fries et al., 2001, 2008). In the three conditions, the relative power decreased with changes in the test-pattern's direction away from preferred (Fig. $7 A, p<10^{-5}$, one-way ANOVA). This was anticipated from the results shown in Figure 4, and reflects the selectivity of the $\gamma$-band signal power for motion direction (Liu and Newsome, 1996). Relative to fixation trials, we found that in both attend-AP in and out conditions the power was suppressed for directions of the test-pattern similar to the preferred (rightmost data points) but enhanced for directions away from the preferred (leftmost data points).

This effect is better illustrated in Figure $7 A$ (right), which shows the average difference in signal power for each comparison and test-pattern direction. The average difference between the attend-AP in and fixation condition (red), and between the attend-AP out and fixation condition (green), shifted from negative to positive values as the direction of the test-pattern deviated from the preferred direction $(p<0.05$, one-way ANOVA). Note that the magnitude of these effects is relatively weak compared with that observed in the lower frequency bands. However, relative to the percentage of signal modulation due to changes in the stimulus direction in the fixation condition (i.e., difference between the signal evoked by the AP + preferred test-pattern and $\mathrm{AP}+90^{\circ}$ away-from-preferred testpattern), the $\gamma$ power in the attend-AP in (or attend-AP out) condition was modulated within a range of $39 \%(26 \%)$ relative to fixation trials. The modulation shifted from 19\% (10\%) suppression (rightmost data point, Fig. 7A, right panel) to $20 \%(16 \%)$ enhancement (leftmost data point).

We have previously suggested that this direction-dependent effect is due to the effects of attention on input signals into area MT (Khayat et al., 2010). Basically, when direction-selective inputs activated by the test stimulus maximally differ in their preferred direction from the attended direction ( $180^{\circ}$ apart, i.e., preferred direction test-pattern vs AP-pattern), they would therefore be maximally suppressed relative to when they become more similar $\left(90^{\circ}\right.$ apart). This would result in the test-pattern direction-dependent modulation shown in Figure 7. If one assumes that LFPs in the $\gamma$ band represent, to a certain extent, the strength of inputs into a local circuit, and therefore into neurons within that circuit, our results would agree with this hypothesis, and with recently proposed computational and theoretical models in which the strength of feature-selective inputs into a neuron is modulated by attention (Reynolds and Heeger, 2009) (see Discussion).

When controlling for the effects of attending to a motion direction by comparing attend-AP in to attend-AP out trials 
A Direction configuration

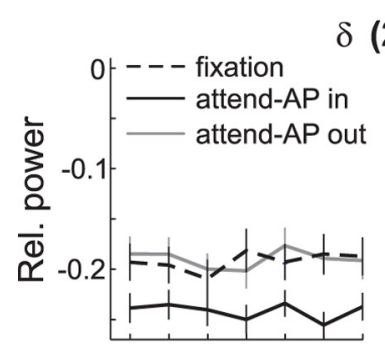

$\delta(2-4 \mathrm{~Hz})$
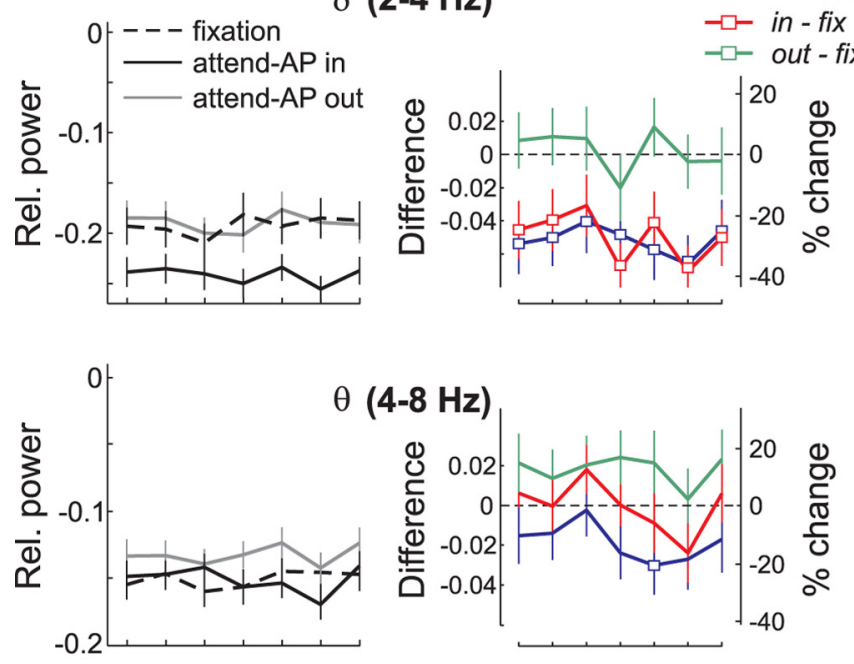

$\theta(4-8 \mathrm{~Hz})$
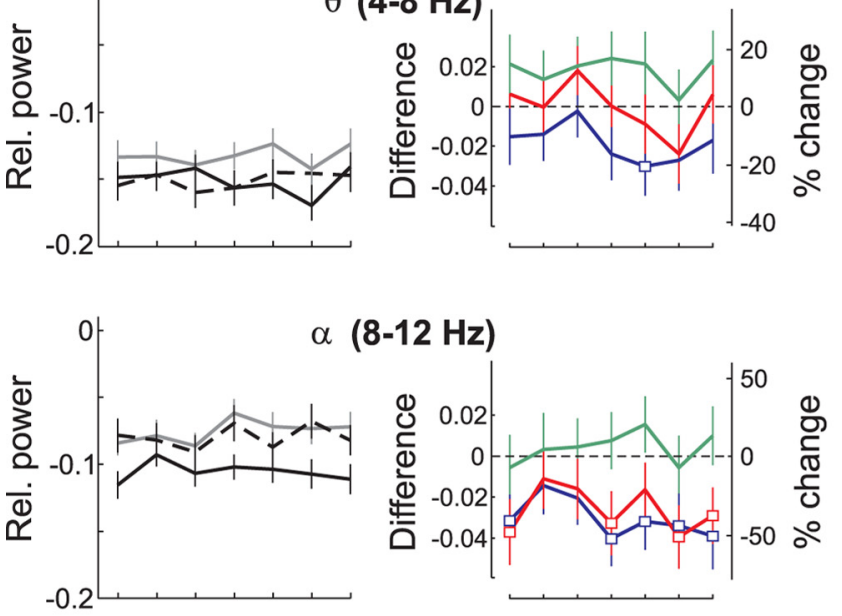

(8-12 Hz)

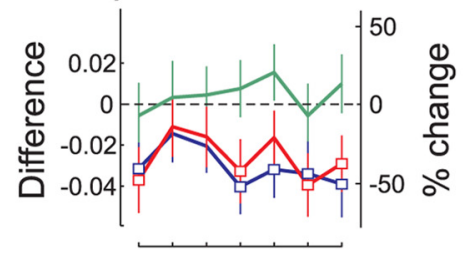

\section{B Contrast configuration \\ $\delta(2-4 \mathrm{~Hz})$}
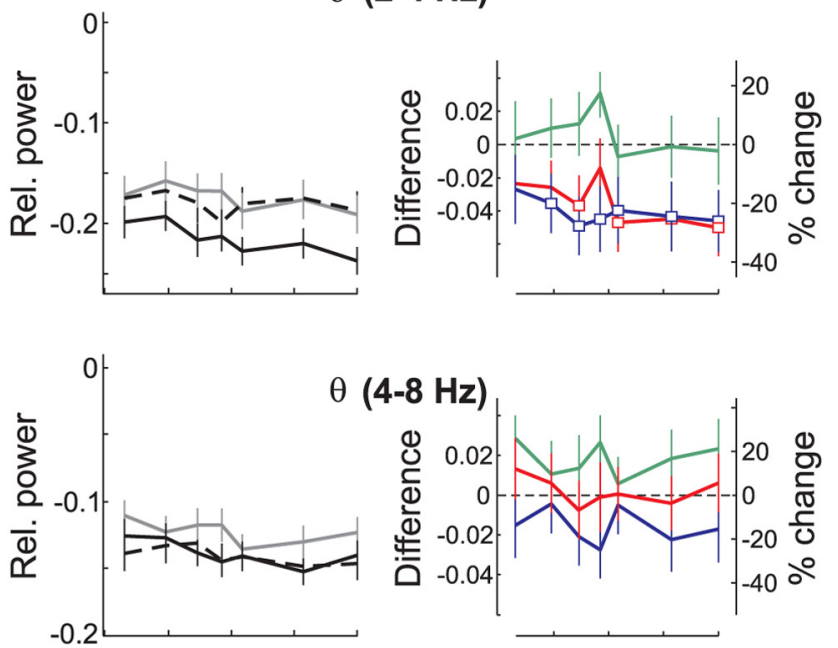

$\theta$ (4-8 Hz)

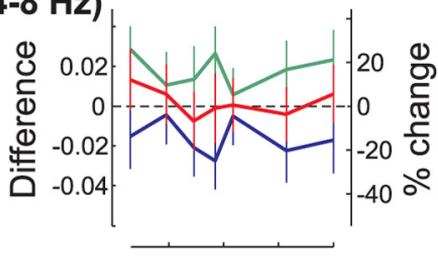

$\alpha(8-12 \mathrm{~Hz})$
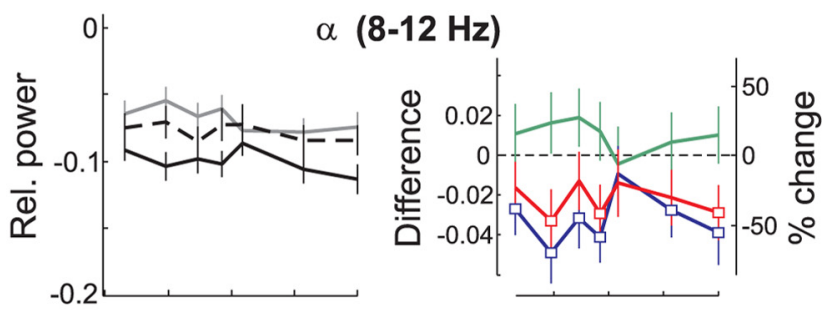

$\beta_{1}(12-20 \mathrm{~Hz})$
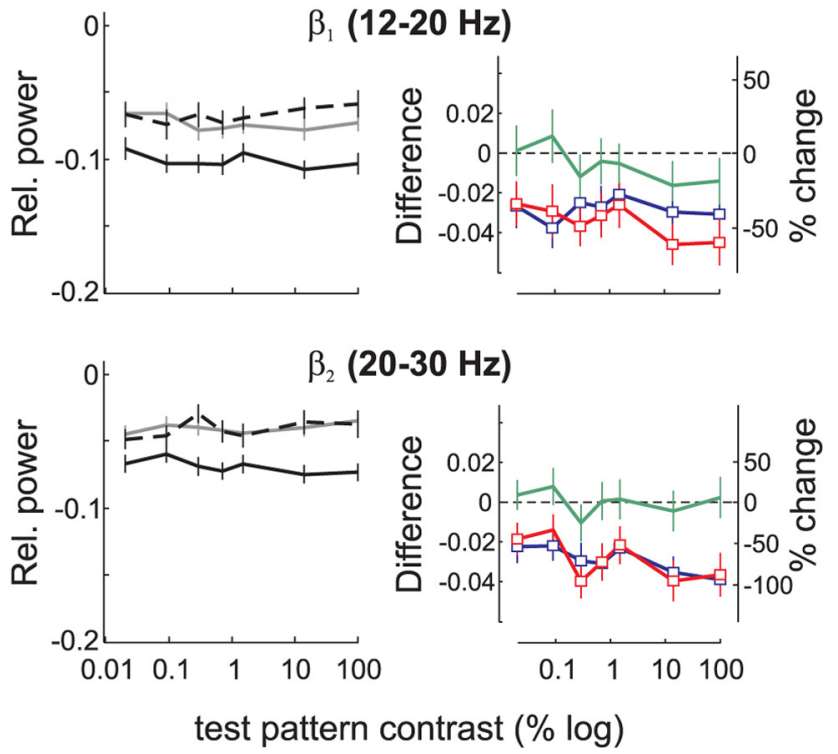

Figure 6. Attentional modulation of LFPs in the low-frequency bands. $\boldsymbol{A}$, Direction configuration data in $\delta, \theta, \alpha, \beta_{1}$ and $\beta_{2}$ frequency bands. The left panels show the mean relative power ( $n=$ 81 ) in the three behavioral conditions for the different test-pattern directions. Black, Attend-AP in; gray, attend-AP out; dashed line, fixation. The right panels show the magnitude of attentional effects expressed as the difference (left axis) and the percentage change (right axis) in power for the comparison attend-AP in versus attend-AP out (in-out, blue), attend-AP in versus fixation (in-fix, red), and attend-AP out versus fixation (out-fix, green). Square data points denote significant differences in power between conditions ( $p<0.05, t$ test). Error bars, SEM. $\boldsymbol{B}$, Contrast configuration data in $\delta, \theta, \alpha, \beta_{1}$ and $\beta_{2}$ frequency bands. Same conventions as in $\boldsymbol{A}$.

(spatial attention), the shift in modulation disappeared, and there was no significant interaction between test-pattern direction and the modulation in the $\gamma$ band (blue, $p>0.8$, one-way ANOVA, Fig. 7A). The difference in $\gamma$ power pooled across all stimulus combinations was significantly lower than zero $(-0.002 \pm 0.001$, mean $\pm \mathrm{SEM}, p<0.05, t$ test $)$, and rep- resented $\sim 6 \%$ mean suppression relative to the magnitude of the tuning, which indicates that the $\gamma$-band LFP-power is generally suppressed when directing attention to the AP-pattern located inside the recorded neuron's RF. These effects of spatial attention, however, did not reach significance when analyzing each stimulus combination separately $(p>0.05, t$ test $)$, and were 


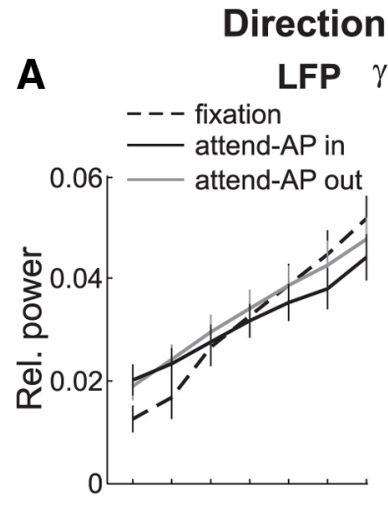

\section{Direction configuration}

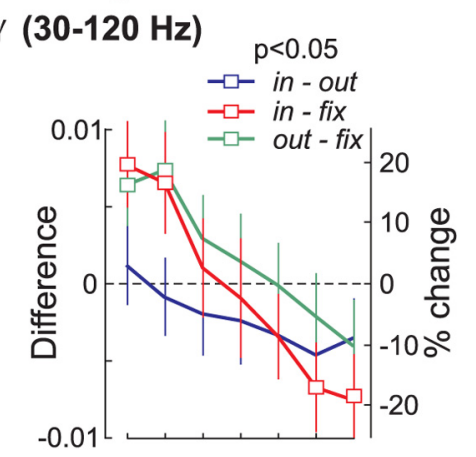

B
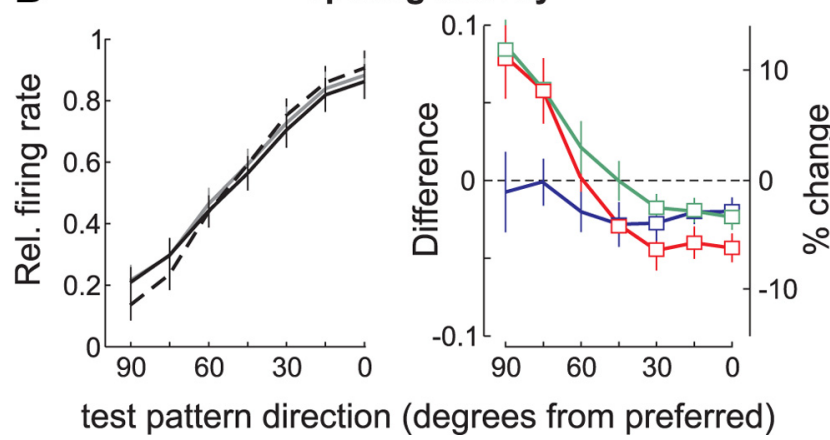

Figure 7. Attentional modulation of LFPs in the $\gamma$ band $(30-120 \mathrm{~Hz})$ and spiking activity, in the direction configuration. The left panels show the relative $\gamma$ power $(\boldsymbol{A})$ and the relative firing rate $(\boldsymbol{B})$ across the population of recording sites $(n=81)$, in the three behavioral conditions. Black, Attend-AP in; gray, attend-AP out; dashed line, fixation. The right panels show the difference (left axis) and the percentage change (right axis) in $\gamma$ power $(\boldsymbol{A})$ and in firing rate $(\boldsymbol{B})$, for the various comparisons. Attend-AP in versus attend-AP out (blue). Attend-AP in versus fixation (red). Attend-AP out versus fixation (green). Squares denote data points with significant differences in power between conditions ( $p<0.05, t$ test). Error bars, SEM.

considerably weaker than those in the corresponding lowfrequency power (Fig. 6A).

\section{Comparison with the spiking activity}

The firing rate modulation between attend-AP in (or attend-AP out), and the fixation condition also shifted from suppression to enhancement as the direction of the test-pattern deviated from preferred (Fig. $7 B$, red and green, right) $\left(p<10^{-6}\right.$, one-way ANOVA). The suppression/enhancement pattern resulted in a total modulation of 16\% (in-fix, red) and 14\% (out-fix, green) change relative to the strength of the tuning. When isolating the effects of spatial attention (attend-AP in vs attend-AP out), the firing rate was generally suppressed when attention was directed to the AP-pattern inside the RF. We found that the difference (blue) was significantly below zero for stimulus pairs in which the test-pattern's direction was more dissimilar to the attended direction (open symbols; $p<0.05, t$ test). For the other test-pattern directions, the suppression was considerably attenuated $(p>$ $0.05, t$ test). There was no significant difference in the modulation as a function of the test-pattern motion direction ( $p>0.5$, oneway ANOVA). A more extensive report of these findings can be found in the work of Khayat et al. (2010). Our interest here is to compare these modulations with the ones of the LFP-signal power shown in Figure 7A.

In summary, the LFP-power modulation in the $\gamma$ band, but not in the other frequency bands, followed the modulation of the neurons spiking activity (Figs. $6 A, 7$ ). In terms of relative
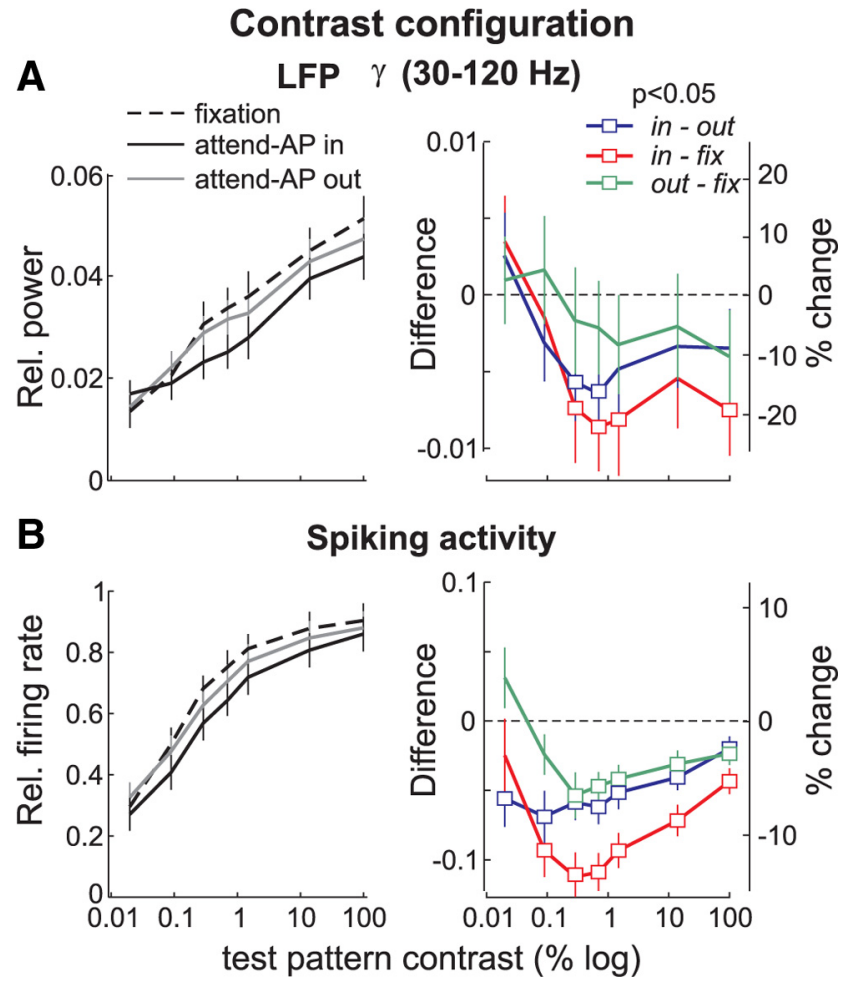

Figure 8. Attentional modulation of LFPs in the $\gamma$ band $(30-120 \mathrm{~Hz})$ and spiking activity, in the contrast configuration. Same conventions as in Figure 7.

strength, the LFP modulation in all frequencies (but the $\theta$ band) was stronger than the modulation of the neurons spiking activity. However, one must interpret this later finding cautiously, mainly because the quantitative details of the way in which LFP signals relate to spiking activity are, so far, poorly understood.

\section{Attentional modulation of LFP signals in the $\gamma$ band: contrast configuration}

In the contrast configuration, we found that the relative power in the $\gamma$ band $(30-120 \mathrm{~Hz})$ was generally suppressed in the attend-AP in compared with the other two conditions (solid black line, Fig. $8 \mathrm{~A}$, left). Moreover, we found that the strength of attentional modulation varied with contrast (red and blue, Fig. $8 \mathrm{~A}$, right); the differences between conditions were the largest for stimuli of intermediate contrast, representing 22\% (in-fix) and $16 \%$ (in-out) change in relative power with respect to the strength of contrast tuning. Note that when comparing the attend-AP out and fixation conditions attentional effects are considerably attenuated, loosing significance (green, $p>0.05, t$ test for each stimulus pair). Importantly, neither for the contrast configuration, nor for the direction configuration (Fig. 7), could these attentional effects reflect changes in the animals' performance since hit rate across all attend-AP in and attend-AP out trials was almost identical, while in fixation trials it was significantly higher (Fig. 2).

\section{Comparison with the spiking activity}

In the contrast configuration, spiking activity was suppressed in both attend-AP in and out conditions, relative to fixation (Fig. $8 \mathrm{~B}$ ). This suppression was significant across most contrast levels (right panel, open symbols, $p<0.01, t$ test). Moreover, this effect was strongest for intermediate contrast stimuli, particularly when comparing the fixation condition to either attended condition 
A

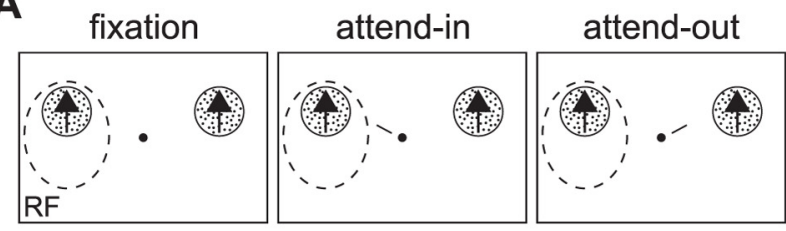

(1) Preferred motion direction

B
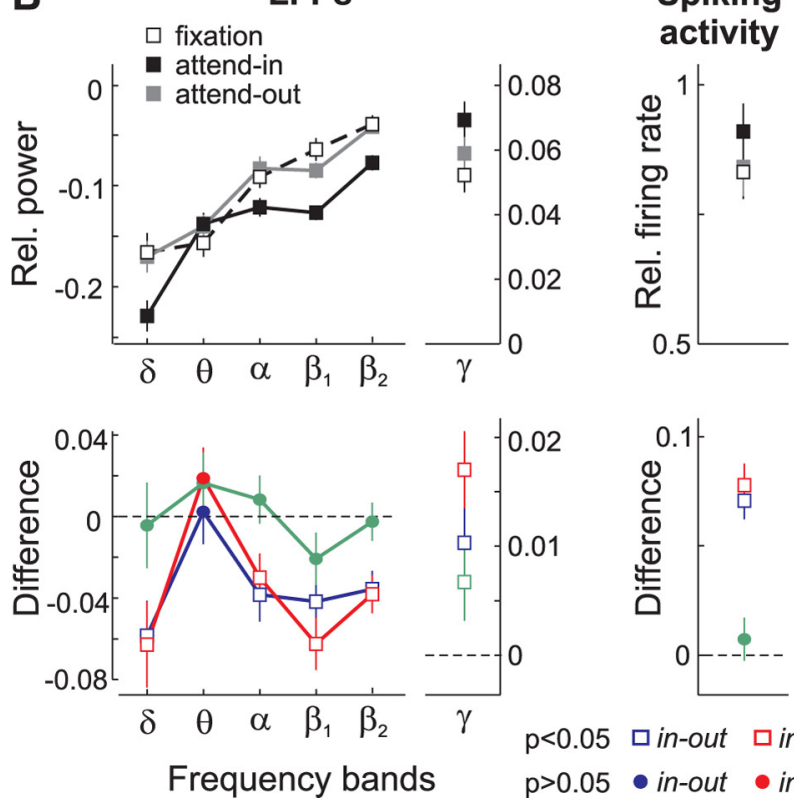

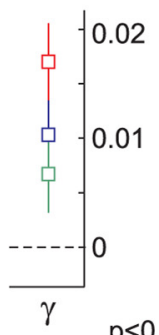

$\mathrm{p}<0.05$ 口in-out $\square$ in-fix $\square$ out-fix

p>0.05 $\bullet$ in-out $\bullet$ in-fix out-fix
C

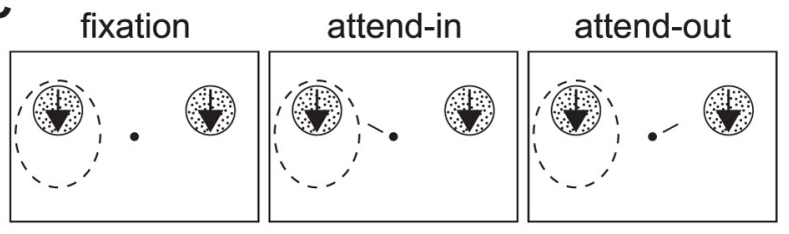

Antipreferred motion direction
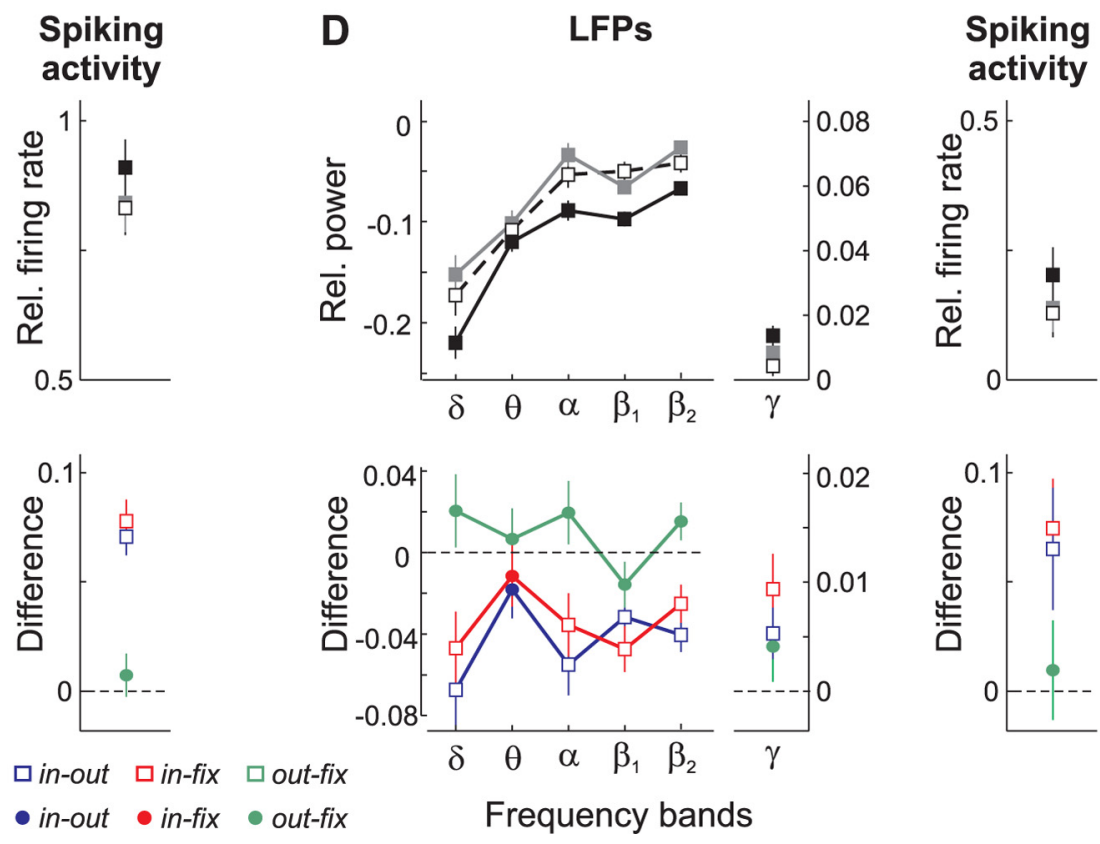

Spiking activity
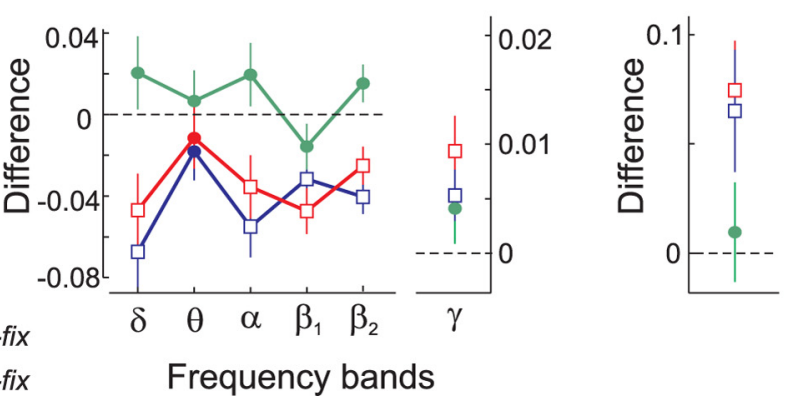

Figure 9. Attentional modulation of population activity $(n=81)$ evoked by either the preferred or antipreferred motion direction. $\boldsymbol{A}$, Stimulus display showing one pattern moving in the preferred direction located inside the RF (dashed circle), and another identical one located in the other hemifield. The animal ignored the patterns (fixation condition) or directed attention to either one of them (attend-in and attend-out conditions). $B$, The top shows the relative power in the $\delta, \theta, \alpha, \beta_{1}, \beta_{2}$ and $\gamma$ bands (left), and relative firing rate (right) during the three conditions with the preferred pattern alone. Black squares, Attend-in; gray squares, attend-out; white squares, fixation. The bottom shows the difference between conditions for each measurement of cortical activity. Blue, Attend-in versus attend-out; red, attend-in versus fixation; green, attend-out versus fixation; open squares denote significant differences in power between conditions ( $p<0.05, t$ test). Circles denote nonsignificant ones ( $p>0.05$ ). Error bars, SEM. C, Stimulus display with one pattern inside the RF moving in the antipreferred direction. $\boldsymbol{D}$, Relative power in the different bands and relative firing rate during the three conditions with the antipreferred pattern alone (top), and the corresponding differences in activity between conditions (bottom). Same conventions as in $\boldsymbol{B}$.

(red and green, $p<0.0005$, one-way ANOVA). Here, the amount of change in the firing rate reached $14 \%$ (in-fix, red) and $8 \%$ (out-fix, green) at intermediate contrast (third data point from the left).

In general, the shape of the modulation of the spiking activity and of the LFP-power in the $\gamma$ band was similar. Although the magnitude of these effects was typically larger in the LFPs relative to the spikes (as it was for the direction configuration), the statistical significance was reached more often when comparing the magnitude of the spiking activity between the different conditions. This may be due to a larger variability in the LFP-power values across individual sites compared with the spikes (see error bars). In the lower frequency bands, the LFP-power modulation did not correlate with the modulation of the spiking activity.

\section{Attentional modulation of LFP-power and spiking activity with one pattern in the RF}

So far, our results show that attending to the AP-pattern could produce either an enhancement or a suppression of both the $\gamma$-band LFP-power, and the neurons firing rate. On the other hand, the power in the $\delta, \alpha, \beta_{1}$, and $\beta_{2}$ band was suppressed when attention was directed to the AP-pattern inside the RF, and was not influenced by the different test-pattern directions or contrast.

To further test whether the suppression of power in these low-frequency bands was due to directing attention into the RF, we used an additional stimulus configuration that differed from the previous ones. During the recording sessions, we included trials in which we presented a single RDP inside the RF and a second identical one in the opposite hemifield (Treue and Martínez Trujillo, 1999). Both patterns moved either in the preferred (Fig. 9A) or antipreferred (AP) direction (Fig. 9C) of the recorded neuron. These trials were randomly interleaved with the trials of the other configurations. The animals were instructed to direct attention either to the pattern located inside (attend-in), or outside (attend-out) the RF, or to the fixation spot (fixation).

Figure $9 B$ shows the LFP-power in the different frequency bands and the spiking activity averaged across recording sites $(n=81)$ during the presentation of the preferred motion direction. When attention was directed inside the recorded neurons' RFs the relative LFP-power (black data points) in the $\delta, \alpha, \beta_{1}$, and $\beta_{2}$ bands, but not in the $\theta$ band, was reduced compared with the fixation (white) and attend-out (gray) condition. The bottom shows the differences between conditions, which were significantly lower than zero (square symbols, $p<0.05, t$ test) in all bands below $30 \mathrm{~Hz}$, except in the $\theta$ band, when comparing attend-in and attend-out trials (blue), as well attend-in and fixation trials (red). On the other hand, the power between attendout and fixation trials did not differ (out-fix, green and circle symbols, $p>0.05, t$ test). 
When the antipreferred pattern was presented in isolation inside the neuron's RF, the LFP-power in these low-frequency bands was modulated in a similar manner (Fig. 9D). These results parallel those observed when two patterns were located inside the neurons' RFs (Fig. 6), and indicate that directing attention into the RF reduces $\delta, \alpha, \beta_{1}$, and $\beta_{2}$ LFP activity in the neuron's vicinity, regardless of the stimulus feature or number of stimuli.

On the other hand, both the LFP-power in the $\gamma$ band and the spiking activity were enhanced during the attend-in condition (black) relative to the other two conditions, when either the preferred (Fig. 9B) or the antipreferred pattern (Fig. 9D) was located in isolation inside the RF. Note that the $\gamma$ power and spiking activity during trials with direction and contrast stimulus pairs (Figs. 7, 8) fall between the level of activity evoked by the preferred pattern alone (Fig. 9B), and the antipreferred pattern alone (Fig. 9D). This shows that in the previous configurations, adding a second test-pattern (with a given direction and contrast) to the AP-pattern inside the RF increased the $\gamma$ power and firing rate, and also reflects the tuning of both measurements for direction and contrast.

When either pattern was presented in isolation inside the RF, the average difference in the $\gamma$ power and spiking activity between attend-in and attend-out or fixation trials (blue and red) was significantly higher than zero (see bottom, square symbols, $p<$ $0.05, t$ test), reflecting an enhancement in both measurements when attention was directed inside the RF (Treue and Martínez Trujillo, 1999). We also isolated the effects of directing attention to a motion direction, while keeping the focus of attention outside the RF (attend-out vs fixation, green data points). We found that the $\gamma$-power and spiking activity evoked by the unattended pattern (preferred or antipreferred) inside the RF was generally enhanced when attention was directed to a similar pattern located outside the RF relative to the fixation condition. However, this direction-dependent effect was relatively weak and reached significance only in the $\gamma$-power activity evoked by the preferred pattern (Fig. $9 B$, green square, $p<0.05, t$ test).

In summary, these results show that directing spatial attention to a single stimulus inside the RF generally enhanced the neurons' spiking activity and the strength of LFP-power in the $\gamma$ band, while consistently decreased the power in $\delta, \alpha, \beta_{1}$, and $\beta_{2}$ bands. We did not find a significant influence of attention on the LFPpower in the $\theta$ band.

\section{Discussion}

Our study demonstrated that: (1) LFP-power in area MT was, in the $\gamma$-frequency range, but not in the $\delta, \theta, \alpha, \beta_{1}$, and $\beta_{2}$ bands, tuned for the motion direction and contrast of moving RDPs, similarly to the neurons firing rate, (2) directing attention into a neuron's RF decreased LFP-power in the bands below $30 \mathrm{~Hz}$ (except the $\theta$ band), and could either increase/decrease power in the $\gamma$ band, (3) directing attention to a motion direction outside the RF had no effect in LFP-power below $30 \mathrm{~Hz}$, but could either increase or decrease $\gamma$ depending on the relationship between the attended direction, and the direction of the stimuli located inside the neurons' RF, d) in the $\gamma$ band, the effects of attention closely matched those observed in the neurons firing rate.

\section{Stimulus tuning of LFPs}

A previous study in area MT has reported tuning of the LFPpower in the $\gamma$ and above frequencies for the direction and speed of moving RDPs (Liu and Newsome, 2006). Our findings agree with this report and also show that the $\gamma$-band power was tuned for stimulus contrast, as previously reported in area V1 (Henrie and Shapley, 2005). Other studies have described stimulus selectivity in this band for orientation, and spatial/temporal frequency in area V1 (Frien et al., 2000; Kayser and König, 2004), and for objects in inferior temporal (IT) cortex (Kreiman et al., 2006). Thus, gamma-band power selectivity for stimulus features is commonly found in visual cortical areas (for review, see Berens et al., 2008).

In our study, the LFP-power in the $\delta, \theta, \alpha, \beta_{1}$, and $\beta_{2}$ bands remained invariant to the different RDPs direction and contrast, which is consistent with previous studies in areas MT (Liu and Newsome, 2006), and V1 (Henrie and Shapley, 2005). However, it apparently differs from a recent report in area V1 showing stimulus tuning in the low frequencies (Katzner et al., 2009). We believe that this discrepancy may be due to the fact that Katzner et al. (2009) used stimuli evoking strong transient neuronal responses, while we purposely avoided using these stimuli and concentrated in analyzing sustained responses, long after stimulus onset.

In accordance with our findings, Belitski et al. (2008) found that area V1 LFP oscillations in frequencies $<40 \mathrm{~Hz}$ show very little signal and noise correlation with frequencies $>40 \mathrm{~Hz}$. They suggested that low-frequency LFPs reflect neural processes that in natural conditions are fully decoupled from those giving rise to spikes and to $\gamma$ LFPs. They further hypothesized that $\gamma$-band LFPs and spikes are generated within the same network. Supporting this idea, a recent study in macaque IT cortex demonstrated that sensory adaptation similarly affects spiking activity and $\gamma$-band LFPs (De Baene and Vogels, 2009). Our results are fully compatible with this hypothesis.

\section{Effects of attention in the $\boldsymbol{\gamma}$ band}

In the direction configuration of the first experiment, shifting attention from the fixation spot to the AP-pattern suppressed both the $\gamma$ band and the spiking activity when the test-pattern moved in the preferred and similar directions. When the testpattern moved in directions orthogonal to the preferred, and therefore closer to the antipreferred, both measurements were enhanced. This result was similar (albeit different in magnitude) regardless of whether the attended AP-pattern was inside, or outside the neurons' RF. We hypothesize that this modulation results from a direction-dependent attentional mechanism that enhances the strength of inputs selective for directions similar to the attended antipreferred direction, while suppresses the strength of inputs selective for more dissimilar directions (Khayat et al., 2010). This agrees with recently proposed models, in which attention modulates the strength of sensory inputs into visual neurons, and consequently the amount of response normalization the cells undergo (Reynolds and Heeger, 2009).

It is reasonable to assume that most direction-selective inputs into MT arise from populations of direction-selective neurons in upstream areas, e.g., V1, V2, or V3 (Born and Bradley, 2005). Since $\gamma$-band LFPs are tuned for direction, one may hypothesize that they represent the contribution of these direction-selective inputs into an MT recording site (Khawaja et al., 2009). The effects of attention in the $\gamma$-band may arise from increases or decreases in the synchronized activity of the corresponding upstream neural populations with RFs at attended locations (Frien et al., 1994; Fries, 2009). Such a mechanism has been proposed as underlying increases in the synchronous firing of V4 neurons with attention (Fries et al., 2001, 2008; Taylor et al., 2005). Other potential explanations are changes in the synchronous behavior of neurons within an MT directional column via interactions with neighboring cells, or with neurons in downstream areas 
through feedback projections (for review, see Tiesinga and Sejnowski, 2009; Gregoriou et al., 2009).

\section{Effects of attention in the low-frequency bands}

One ubiquitous finding across all stimulus configurations used in this study was that directing attention into the neurons' RF suppressed LFP-power in the $\delta, \alpha, \beta_{1}$, and $\beta_{2}$ bands, but not in the $\theta$ band. This effect was independent of the stimulus configuration (one- or two-stimuli in the RF), and disappeared when the animals switched attention between the fixation spot and the pattern outside the RF (attending to a direction). Importantly, our experimental manipulations led to situations in which spatial attention either decreased (contrast configuration), or increased (single pattern inside the RF configuration) the firing rate of an MT neuron when directed into its RF. In all these situations, however, LFP-power in the $\delta, \alpha, \beta_{1}$, and $\beta_{2}$ bands was suppressed, suggesting that this effect was not linked to increases or decreases in firing rate. Moreover, the magnitude of the modulation in these bands was stronger than in the $\gamma$ band.

A study in macaque area V4 using a "one stimulus inside and one outside the RF" configuration (Fries et al., 2008), as well as EEG/MEG studies in humans (Worden et al., 2000; Kelly et al., 2006, 2009; Thut et al., 2006; Siegel et al., 2008), reported similar results. However, Lakatos et al. (2008) reported in area V1 that attention could also increase the power of low-frequency oscillations in tasks where the occurrence of a stimulus within a rhythmic stream is highly predictable. They suggested that attention could increase or decrease $\delta$ power in such a manner that a phase of high excitability would coincide with the stimulus occurrence; i.e., enforcing oscillatory entrainment to a task-relevant input stream. On the other hand, when the behaviorally relevant event is unpredictable in time-as was the direction change of our experiment-attention operates best in a "continuous mode" characterized by extended increase in $\gamma$ and suppression of lowfrequency oscillations (Schroeder and Lakatos, 2009). The results of Lakatos et al. (2008), together with the ones of Katzner et al. (2009), suggest that LFP-power in the low frequencies can also vary according to the nature of the sensory input and the task (see also Buschman and Miller, 2009).

Interestingly, despite the observed effects of attending to a stimulus direction outside the RF on firing rate and $\gamma$ LFPs, we did not find any correlate of such effects in the low frequencies. A previous study in area V4 (Bichot et al., 2005) reported clear enhancements in firing rate and synchrony between spikes and $\gamma$-band activity when attending to a stimulus feature, but no effect in the low frequencies. These results suggest that, unlike spatial attention, feature-dependent attentional mechanisms may not "target" low-frequency oscillations.

One possible explanation for the latter phenomenon is linked to the proposal that low-frequency LFPs reflect signal pooling (through lateral connections) across neurons within a cortical region when feedforward inputs are weak, favoring the detection of low intensity visual signals (Nauhaus et al., 2009). If spatial attention increases the strength of feedforward (upstream) inputs to neurons with RFs at the attended location (Reynolds et al., 2000; Martínez-Trujillo and Treue, 2002; Reynolds and Heeger, 2009; Khayat et al., 2010), it will decrease the need for signal pooling. Because inputs' strength and low-frequency power are inversely correlated, one would expect a decrease in low-frequency oscillations in the region targeted by the strengthened inputs. On the other hand, because more global featuredependent mechanisms produce, within the same cortical region, increases and decreases in inputs' strength, depending on the selectivity of the inputs (i.e., inputs selective for the attended feature will be enhanced while inputs selective for dissimilar features will be suppressed), its net effect on inputs' strength and low-frequency power within that region would be "null" or very small.

A puzzling finding in our experiments was the lack of attentional modulation in the $\theta$ band. Previous studies found that the phase of low-frequency oscillations modulates the strength of $\gamma$-band synchronization (Lakatos et al., 2005; Canolty et al., 2006), and it has been suggested that the cross-frequency phase synchronization between $\theta$ and $\gamma$ oscillations play a fundamental role in memory processes (Sauseng et al., 2009, 2010), and learning (Tort et al., 2009). It is possible that in our scenario the power in the $\theta$ band was "preserved" by the attentional mechanism to facilitate the implementation of these task components. This hypothesis, however, remains to be tested.

\section{References}

Belitski A, Gretton A, Magri C, Murayama Y, Montemurro MA, Logothetis NK, Panzeri S (2008) Low-frequency local field potentials and spikes in primary visual cortex convey independent visual information. J Neurosci 28:5696-5709.

Berens P, Keliris GA, Ecker AS, Logothetis NK, Tolias AS (2008) Feature selectivity of the gamma-band of the local field potential in primate primary visual cortex. Front Neurosci 2:199-207.

Bichot NP, Rossi AF, Desimone R (2005) Parallel and serial neural mechanisms for visual search in macaque area V4. Science 308:529-534.

Born RT, Bradley DC (2005) Structure and function of visual area MT. Annu Rev Neurosci 28:157-189.

Buschman TJ, Miller EK (2009) Serial, covert shifts of attention during visual search are reflected by the frontal eye fields and correlated with population oscillations. Neuron 63:386-396.

Busse L, Katzner S, Treue S (2008) Temporal dynamics of neuronal modulation during exogenous and endogenous shifts of visual attention in macaque area MT. Proc Natl Acad Sci U S A 105:16380-16385.

Buzsáki G (2002) Theta oscillations in the hippocampus. Neuron 33:325-340.

Buzsáki G (2006) Rhythms of the brain. New York: Oxford UP.

Canolty RT, Edwards E, Dalal SS, Soltani M, Nagarajan SS, Kirsch HE, Berger MS, Barbaro NM, Knight RT (2006) High gamma power is phaselocked to theta oscillations in human neocortex. Science 313:1626-1628.

De Baene W, Vogels R (2009) Effects of adaptation on the stimulus selectivity of macaque inferior temporal spiking activity and local field potentials. Cereb Cortex. Advance online publication. Retrieved December 27, 2009. doi:10.1093/cercor/bhp277.

Felleman DJ, Kaas JH (1984) Receptive-field properties of neurons in middle temporal visual area (MT) of owl monkeys. J Neurophysiol 52:488-513.

Frien A, Eckhorn R, Bauer R, Woelbern T, Kehr H (1994) Stimulus-specific fast oscillations at zero phase between visual areas V1 and V2 of awake monkey. Neuroreport 5:2273-2277.

Frien A, Eckhorn R, Bauer R, Woelbern T, Gabriel A (2000) Fast oscillations display sharper orientation tuning than slower components of the same recordings in striate cortex of the awake monkey. Eur J Neurosci 12:1453-1465.

Fries P (2009) Neuronal gamma-band synchronization as a fundamental process in cortical computation. Annu Rev Neurosci 32:209-224.

Fries P, Reynolds JH, Rorie AE, Desimone R (2001) Modulation of oscillatory neuronal synchronization by selective visual attention. Science 291:1560-1563.

Fries P, Womelsdorf T, Oostenveld R, Desimone R (2008) The effects of visual stimulation and selective visual attention on rhythmic neuronal synchronization in macaque area V4. J Neurosci 28:4823-4835.

Gregoriou GG, Gotts SJ, Zhou H, Desimone R (2009) High-frequency, long-range coupling between prefrontal and visual cortex during attention. Science 324:1207-1210.

Henrie JA, Shapley R (2005) LFP power spectra in V1 cortex: the graded effect of stimulus contrast. J Neurophysiol 94:479-490.

Katzner S, Nauhaus I, Benucci A, Bonin V, Ringach DL, Carandini M (2009) Local origin of field potentials in visual cortex. Neuron 61:35-41. 
Kayser C, König P (2004) Stimulus locking and feature selectivity prevail in complementary frequency ranges of V1 local field potentials. Eur J Neurosci 19:485-489.

Kelly SP, Lalor EC, Reilly RB, Foxe JJ (2006) Increases in alpha oscillatory power reflect an active retinotopic mechanism for distracter suppression during sustained visuospatial attention. J Neurophysiol 95:3844-3851.

Kelly SP, Gomez-Ramirez M, Foxe JJ (2009) The strength of anticipatory spatial biasing predicts target discrimination at attended locations: a highdensity EEG study. Eur J Neurosci 30:2224-2234.

Khawaja FA, Tsui JM, Pack CC (2009) Pattern motion selectivity of spiking outputs and local field potentials in macaque visual cortex. J Neurosci 29:13702-13709.

Khayat PS, Spekreijse H, Roelfsema PR (2006) Attention lights up new object representations before the old ones fade away. J Neurosci 26:138-142.

Khayat PS, Niebergall R, Martinez-Trujillo JC (2010) Attention differentially modulates similar neuronal responses evoked by varying contrast and direction stimuli in area MT. J Neurosci 30:2188-2197.

Kreiman G, Hung CP, Kraskov A, Quiroga RQ, Poggio T, DiCarlo JJ (2006) Object selectivity of local field potentials and spikes in the macaque inferior temporal cortex. Neuron 49:433-445.

Kruse W, Eckhorn R (1996) Inhibition of sustained gamma oscillations $(35-80 \mathrm{~Hz})$ by fast transient responses in cat visual cortex. Proc Natl Acad Sci U S A 93:6112-6117.

Lakatos P, Shah AS, Knuth KH, Ulbert I, Karmos G, Schroeder CE (2005) An oscillatory hierarchy controlling neuronal excitability and stimulus processing in the auditory cortex. J Neurophysiol 94:1904-1911.

Lakatos P, Karmos G, Mehta AD, Ulbert I, Schroeder CE (2008) Oscillatory entrainment as a mechanism of attentional selection. Science 320:110 113.

Leopold DA, Murayama Y, Logothetis NK (2003) Very slow activity fluctuations in monkey visual cortex: implications for functional brain imaging. Cereb Cortex 13:422-433.

Liu J, Newsome WT (2006) Local field potential in cortical area MT: stimulus tuning and behavioral correlations. J Neurosci 26:7779-7790.

Logothetis NK (2003) The underpinnings of the BOLD functional magnetic resonance imaging signal. J Neurosci 23:3963-3971.

Logothetis NK, Wandell BA (2004) Interpreting the BOLD signal. Annu Rev Physiol 66:735-769.

Martínez-Trujillo J, Treue S (2002) Attentional modulation strength in cortical area MT depends on stimulus contrast. Neuron 35:365-370.

Martinez-Trujillo JC, Treue S (2004) Feature-based attention increases the selectivity of population responses in primate visual cortex. Curr Biol 14:744-751.

Mitzdorf U (1985) Current source-density method and application in cat cerebral cortex: investigation of evoked potentials and EEG phenomena. Physiol Rev 65:37-100.

Mitzdorf U (1987) Properties of the evoked potential generators: current source-density analysis of visually evoked potentials in the cat cortex. Int J Neurosci 33:33-59.

Nauhaus I, Busse L, Carandini M, Ringach DL (2009) Stimulus contrast modulates functional connectivity in visual cortex. Nat Neurosci 12:70-76.

Reynolds JH, Heeger DJ (2009) The normalization model of attention. Neuron 61:168-185.

Reynolds JH, Pasternak T, Desimone R (2000) Attention increases sensitivity of V4 neurons. Neuron 26:703-714.

Sauseng P, Klimesch W, Heise KF, Gruber WR, Holz E, Karim AA, Glennon M, Gerloff C, Birbaumer N, Hummel FC (2009) Brain oscillatory substrates of visual short-term memory capacity. Curr Biol 19:1846-1852.

Sauseng P, Griesmayr B, Freunberger R, Klimesch W (2010) Control mechanisms in working memory: a possible function of EEG theta oscillations. Neurosci Biobehav Rev 34:1015-1022.

Schroeder CE, Lakatos P (2009) The gamma oscillation: master or slave? Brain Topogr 22:24-26.

Sclar G, Maunsell JH, Lennie P (1990) Coding of image contrast in central visual pathways of the macaque monkey. Vision Res 30:1-10.

Seidemann E, Newsome WT (1999) Effect of spatial attention on the responses of area MT neurons. J Neurophysiol 81:1783-1794.

Siegel M, Donner TH, Oostenveld R, Fries P, Engel AK (2008) Neuronal synchronization along the dorsal visual pathway reflects the focus of spatial attention. Neuron 60:709-719.

Taylor K, Mandon S, Freiwald WA, Kreiter AK (2005) Coherent oscillatory activity in monkey area $\mathrm{v} 4$ predicts successful allocation of attention. Cereb Cortex 15:1424-1437.

Thut G, Nietzel A, Brandt SA, Pascual-Leone A (2006) Alpha-band electroencephalographic activity over occipital cortex indexes visuospatial attention bias and predicts visual target detection. J Neurosci 26:9494-9502.

Tiesinga P, Sejnowski TJ (2009) Cortical enlightenment: are attentional gamma oscillations driven by ING or PING? Neuron 63:727-732.

Tort AB, Komorowski RW, Manns JR, Kopell NJ, Eichenbaum H (2009) Theta-gamma coupling increases during the learning of item-context associations. Proc Natl Acad Sci U S A 106:20942-20947.

Treue S, Maunsell JH (1996) Attentional modulation of visual motion processing in cortical areas MT and MST. Nature 382:539-541.

Treue S, Martínez Trujillo JC (1999) Feature-based attention influences motion processing gain in macaque visual cortex. Nature 399:575-579.

Worden MS, Foxe JJ, Wang N, Simpson GV (2000) Anticipatory biasing of visuospatial attention indexed by retinotopically specific alphaband electroencephalography increases over occipital cortex. J Neurosci 20:RC63(1-6).

Zeki S (1980) The response properties of cells in the middle temporal area (area MT) of owl monkey visual cortex. Proc R Soc Lond B Biol Sci 207:239-248. 